18th Annual International Conference of the IEEE Engineering in Medicine and Biology Society, Amsterdam 1996 4.2.7: Time-frequency Analysis of Various Signals I - Wavelet Transforms

\title{
DETECTION AND QUANTIFICATION OF VENOUS AIR EMBOLISM BY WAVELET ANALYSIS OF DOPPLER HEART SOUND
}

\author{
Francis H.Y. Chan', Brent C.B. Chan ${ }^{1}$, F.K. Lam ${ }^{1}$, Paul W.F. Poon ${ }^{2}$, P.W. Lui ${ }^{3}$ and H. Wang ${ }^{3}$ \\ ${ }^{1}$ fhychan, cbchan, fklam@hkueee.hku.hk, ${ }^{2}$ ppoon@mail.ncku.edu.tw, ${ }^{3}$ pwlui, hwang@mail.vghtpe.gov.tw

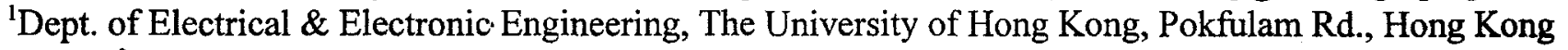 \\ ${ }^{2}$ Dept. of Physiology, National Cheng Kung University, 1 University Rd., Tainan, Taiwan \\ ${ }^{3}$ Dept. of Anesthesiology, Veterans General Hospital-Taipei, 201 Shih-pai Rd. Sec. 2, Taipei, Taiwan
}

\begin{abstract}
The wavelet analysis of the Doppler heart sound detected under controlled venous air embolism at sub-clinically and clinically significant volumes was studied in anaesthetized dogs. Signal processing with wavelet enhances the power of embolic signal and facilitates the simple detection and extraction of embolic heart beats by thresholding. The cumulative power of the extracted embolic heart beats is found to be linearly related to the volume of embolic air on the log-log scale, suggesting that it is feasible to estimate clinically significant volume of embolic air in human subjects by linearly extrapolating from sub-clinical embolic volumes.
\end{abstract}

\section{INTRODUCTION}

Venous air embolism (VAE) is potentially hazardous during surgery where air fills the incised veins by negative pressure. Ultrasonic precordial Doppler monitoring is one of the most sensitive and non-invasive methods for detecting VAE [1-3]. However, it requires the constant attention of an anaesthetist to listen to subtle changes in the Doppler heart sound (DHS). Besides, it cannot quantify the amount of air entering circulation. Such information is essential for the anaesthetist to prescribe suitable clinical treatments [4]. In this study, we thus applied the wavelet analysis to the DHS signal in anaesthetized dogs under simulated conditions of sub-clinically and clinically significant VAE with a view to detecting VAE and quantifying the embolic air.

\section{MATERIALS AND METHODS}

Three anaesthetized mongrel dogs weighing approximately $15 \mathrm{~kg}$ were placed in supine position with their lungs being mechanically ventilated. Intravenous bolus air infusion $(0.05$, $0.10,0.20,0.40,0.80$ and $1.60 \mathrm{ml}$ ) and continuous air infusion $(0.05,0.10,0.20$ and $0.40 \mathrm{ml} / \mathrm{kg} / \mathrm{min}$ for $5 \mathrm{~min}$ ) were conducted through the external right jugular vein. Preinfusion baseline period $(5 \mathrm{~min})$ and appropriate resting period in between successive infusions ( 5 to $10 \mathrm{~min}$ ) were allowed. The DHS and ECG (lead ID) signals during the whole process were recorded on an audio-tape recorder. Signals were later digitized at $4 \mathrm{kHz}, 16$-bit resolution on a Pentium-based multimedia $\mathrm{PC}$ for offline processing.
The DHS signal was partitioned into individual heart beats for systematic analysis using the ECG as a consistent timing signal. The local minima of the moving average [5] of the full-wave rectified ECG were treated as the boundaries of individual heart beats (Fig. 1). The partitioned DHS signal was then processed using the Daubechies 20-coefficient wavelet [6]. The variation of the signal power of individual heart beats (beat power for short) of the original and waveletprocessed DHS under different volumes of air infusion was studied. These two sets of beat power data were normalized with respect to the mean power of the pre-infusion heart beats of the original and wavelet-processed DHS respectively for the sake of comparison.

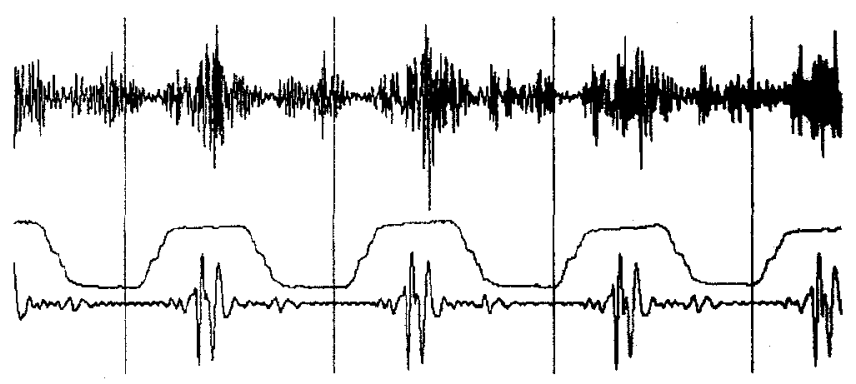

Fig. 1. Partition of DHS (upper tracing) into individual heart beats using the local minima (indicated by the vertical lines) of the moving average (middle tracing) of the ECG (lower tracing) as the boundaries.

\section{RESULTS}

The wavelet-processed DHS signal showed striking performance in distinguishing embolic heart beats (EHB) from the normal (Fig. 2). Essentially, the contrast of the embolic to normal beat power was greatly enhanced by the wavelet analysis due to the extra high-frequency components of the EHB and the band-pass nature of the wavelet. Hence, the EHB could be detected and extracted simply by thresholding (Fig. 3). We summed the beat power of all the extracted EHB for each volume of air infusion and called it the cumulative embolic power (CEP) at such volume. A linear relationship was obtained between the CEP and the volume of infused air on the log-log scale (Fig. 4). 


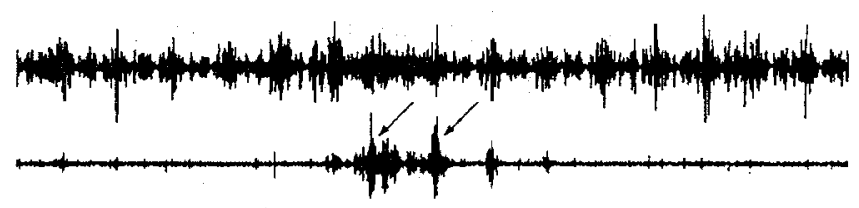

Fig. 2. The original (upper tracing) and wavelet-processed (lower tracing) DHS at $0.40 \mathrm{ml}$ air infusion of Dog 2. The EHB are marked by arrows and confirmed by an experienced anaesthetist who listened to the playback signal of the recorded DHS.

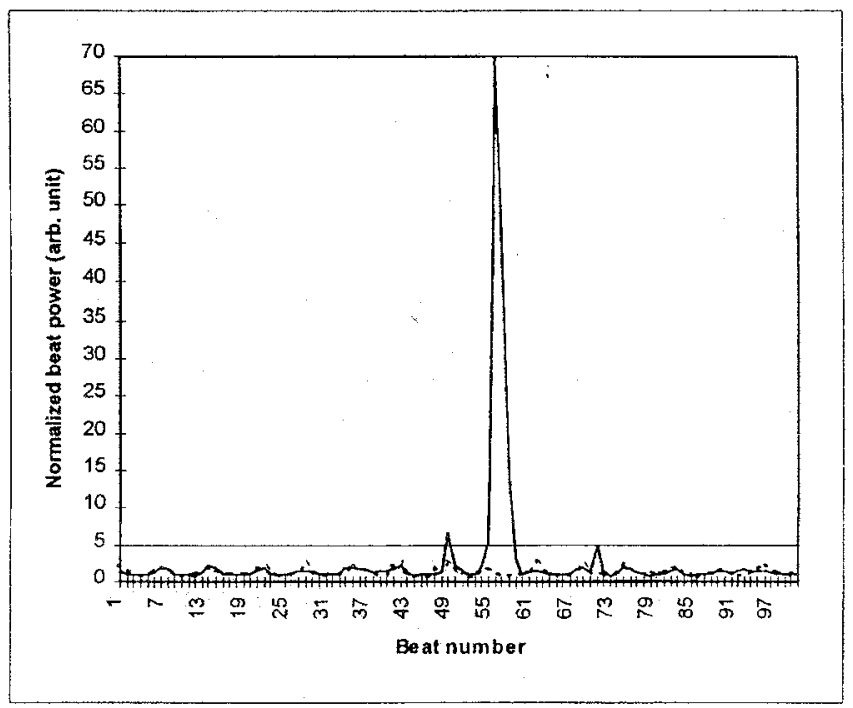

Fig. 3. Plot of the normalized beat power against the beat number at $0.40 \mathrm{ml}$ air infusion of Dog 2 (broken line for original and solid line for wavelet-processed DHS). A threshold of 5 (indicated by the horizontal line) can reliably detect and extract the EHB. The periodic ripples reflect the noise of the mechanical ventilator.

\section{CONCLUSIONS}

The wavelet analysis of the DHS signal can greatly enhance the contrast of the embolic to normal beat power, facilitating the simple detection and extraction of EHB by thresholding. This feature is useful for the design of an automatic system for detecting VAE [7]. Besides, the linear relationship between the CEP and the volume of infused air shows the feasibility of estimating clinically significant volume of embolic air by extrapolating the linear trend obtained during sub-clinical VAE for a subject. Finally, our method is applicable even when the DHS is embedded in the noise of the mechanical ventilator.

(Supported in part by the University of Hong Kong Research Grants and National Science Council, Taiwan.)

\section{REFERENCES}

[1] D. Michenfelder, R.H. Miller and G.A. Gronert, "Evaluation of an ultrasonic device (Doppler) for the diagnosis of venous air embolism," Anesthesiology, vol. 36, pp. 164-167, 1972.

[2] B. English, D. Westenskow, M.R. Hodges and T.H. Stanley, "Comparison of venous air embolism monitoring methods in supine dogs," Anesthesiology, vol. 48 , pp. $425-429,1978$.

[3] M. Malinow, J.S. Naulty, C.O. Hunt, S. Datta and G.W. Ostheimer, "Precordial ultrasonic monitoring during cesarean delivery," Anesthesiology, vol. 66, pp. 816-819, 1987.

[4] R.C. Shupax, "Air embolism and its influence on anesthetic management," Current Reviews in Clinical Anesthesia, vol. 11, pp. 113-124, 1991.

[5] A.V. Oppenheim and R.W. Schafer, "Discrete-time signal processing," Prentice-Hall, 1989.

[6] I. Daubechies, "Ten lectures on wavelets," SIAM, 1992.

[7] B.C.B. Chan, F.H.Y. Chan, F.K. Lam, P.W. Lui and P.W.F. Poon, "Real-time monitoring of venous air embolism with Doppler heart sound using multiresolution wavelet analysis," Engineering and Physics in Medicine, Queenstown, New Zealand (Conference Proceedings), pp. 185, Nov. 1995.

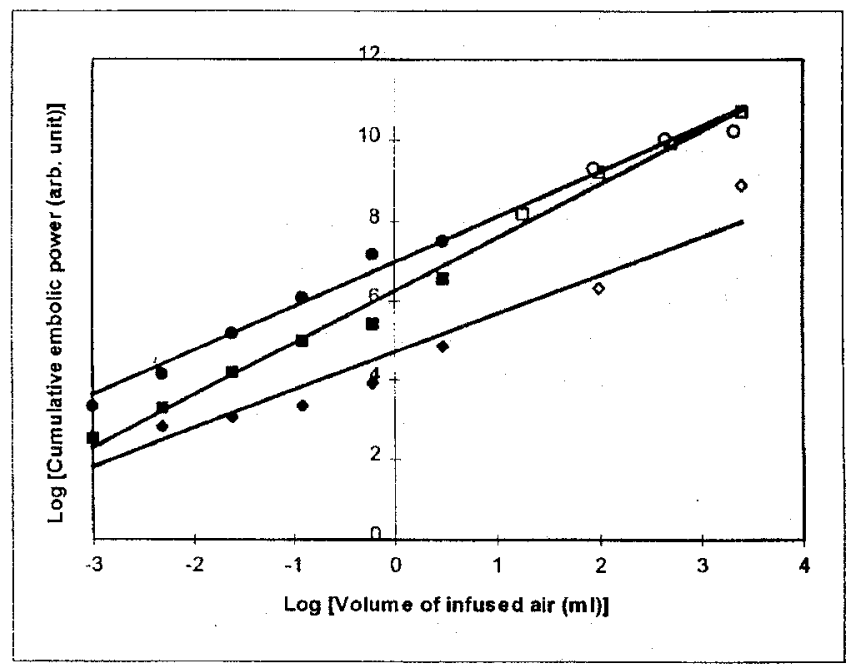

Fig. 4. Log-log plot (natural log) of the cumulative embolic power against the volume of infused air. Filled markers for bolus infusion while empty markers for continuous infusion. Results of linear regression and correlation coefficient: Dog 1 ( ) $y=0.97 x+4.73, r=0.964 ; \operatorname{Dog} 2(\mathbf{m}) y=1.33 x+6.27$, $r=0.996 ; \operatorname{Dog} 3(\bullet) y=1.13 x+6.97, r=0.994$. Some data points $(0.05 \mathrm{ml} / \mathrm{kg} / \mathrm{min}$ for Dogs $1 \& 3$ and $0.20 \mathrm{ml} / \mathrm{kg} / \mathrm{min}$ for Dog 1) were discarded due to uncontrolled interference in experimentation. 